\title{
Excitation of localized wave packet in swept-wing supersonic boundary layer
}

\author{
Aleksey Yatskikh $^{1,2, *}$, Marina Rumenskikh ${ }^{1,2}$, Yury Yermolaev ${ }^{1}$, Aleksander Kosinov $^{1,2}$ and \\ Nikolay Semionov ${ }^{1}$ \\ ${ }^{1}$ Khristianovich Institute of Theoretical and Applied Mechanics SB RAS, 630090 Novosibirsk, Russia \\ ${ }^{2}$ Novosibirsk State University, 630090 Novosibirsk, Russia
}

\begin{abstract}
The evolution of the artificial wave packet in swept-wing supersonic boundary layer was experimentally studied at $M=2$. The localized disturbances were generated by a pulse glow discharge. Measurements were provided by a hot-wire anemometer. The spatial structure of the wave packet was studied. It was found that the wave packet has an asymmetric shape. In addition, the velocity of the propagation downstream of the wave packet was estimated.
\end{abstract}

\section{Introduction}

To solve the problem of laminarization the detailed knowledge of laminar-turbulent transition mechanisms is required. At present it is generally recognized that the onset of turbulence is connected with the loss of stability of the initial laminar flow.

The study of the evolution of artificially controlled perturbations allows finding out the wave characteristics of the boundary layers. A commonly used method of the actuation of controlled perturbations in a supersonic boundary layer is a high-frequency electric glow discharge. The experimental confirmation of the main concepts of the linear theory of stability in supersonic zero-pressure-gradient flow on a flat plate was obtained by this method in [1]. Also, this approach is useful in studies of laminar-turbulent transition in inhomogeneous and 3D supersonic boundary layers [2-4]. The approach of periodical disturbances is also used in direct numerical simulation studies (DNS). In [5, 6] results coinciding with experiments were obtained. Also, the method of artificial excitation of periodically pulsations in boundary layers is efficiently used at the hypersonic speeds [7-9].

However, the latter method allows us to study the evolution of narrowband disturbances while natural laminar-turbulent transition is associated with the evolution downstream of fluctuations consisted of a wide range of frequencies and wavenumbers excited by freestream turbulence. Therefore the study of the evolution of broadband disturbance (wave packets) is promising.

The first experimental studies of wave packet propagation in boundary layers were provided at low speed of flow [10]. The evolution of wave packets generated by a shortduration acoustic pulse was investigated by hot-wire measurements. These measurements showed good agreement with the results obtained from the theoretical model which

* Corresponding author: 73.yatskikh@gmail.com 
represented the wave packet as a superposition of individual disturbances for all frequencies and spanwise wave numbers of the most unstable linear waves [11]. Further development of the approach of wave packets led to significant progress in understanding the processes of laminar-turbulent transition at a subsonic speed. Thus, e.g. in [12] the emergence of wave packets, or forerunners, in the frontal regions of localized structures and their evolution in subsonic boundary layer was experimentally examined. The experiments showed that the forerunners were Tollmien-Schlichting wave packets.

At high speeds of flows the method of the excitation of artificial wave packets is actively developing. The evolution of wave packets in the hypersonic boundary layer of a nozzle was experimentally studied in [13]. The measurements were taken along the axial line of the nozzle using surface pressure transducers. The linear and non-linear growth of wave packets and their transformation into a turbulent spot were found. The evolution and transformation into turbulent spots of wave packets are shown as a continuous process. Also, the method of wave packets is widely used in numerical study of laminar-turbulent transition [14-16].

In [17-19] the technique of pulsed excitation of wave formations was developed and used to study the turbulence origin in a two-dimensional supersonic boundary layer. The evolution of the artificial wave packet in the boundary layer on a flat plate at $M=2$ was studied in these works with the help of hot-wire measurements. The wave packets were generated by a pulse discharge. The analysis of the experimental data has shown that for different frequency modes the most unstable waves are oblique. This agrees with the theoretical results.

The main goal of the present work is to carry out the experimental study of the evolution of wave packets in a three-dimensional supersonic boundary layer.

\section{Experimental setup}

Experiments were performed in the T-325 long-duration blowdown low-noise supersonic wind tunnel, at the Khristianovich Institute of Theoretical and Applied Mechanics SB RAS, at Mach number $M=2$ and unit Reynolds number $\operatorname{Re}_{1}=6 \cdot 10^{6} \mathrm{~m}^{-1}$. A model of swept-wing with a sharp leading edge and a source of controlled disturbances were used (Fig. 1). The swept angle of the leading edge is $40^{\circ}$. The model was installed with zero angle of attack.

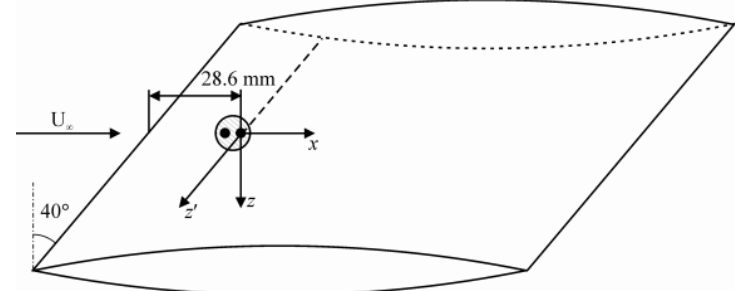

Fig. 1. Swept-wing model with a source of controlled perturbations.

To initiate controlled wave packets an electric discharge was ignited between two copper electrodes separated from each other and the model with an insulator. The insulator and electrodes were mounted flush with the model surface. The electrode centers were located parallel to the direction of the flow (the $x$-axis). The electrode diameter was $0.6 \mathrm{~mm}$, while the minimum distance between them was approximately $0.6 \mathrm{~mm}$. Highvoltage $(\sim 1 \mathrm{kV})$ pulses were supplied to the electrodes from the ignition scheme.

The ignition scheme for pulsed electric discharges is based on disrupting the current in the primary circuit of the ignition coil with a powerful bipolar transistor. Disrupting the current in the primary circuit of the ignition coil generates high-voltage pulsing. 
In these experiments the duration of discharge was about $20 \mu \mathrm{s}$. The energy of a single discharge was about $0.12 \mathrm{~mJ}$.

Pulsations in a supersonic flow were measured using constant-temperature hot-wire anemometry (CTA). A hot-wire probe made of a tungsten wire with a $10 \mu \mathrm{m}$ diameter was used. The overheat rate of the hot-wire probe was set to 0.8 , while perturbations measured by CTA were predominantly mass flux pulsations $\left(m^{\prime}\right)$. The measurements were performed at the layer with the most intense natural pulsations of the boundary layer. The probe was moved with the help of traversing gears which the wind tunnel was equipped with. The positioning accuracy of the probe was about $0.1 \mathrm{~mm}$.

To separate perturbations generated by the discharge from the background natural fluctuations of the boundary layer, the measurements were synchronized with discharge ignition. 320 traces were recorded at each measurement point. Data processing included ensemble-averaging 320 traces.

\section{Results}

The isolines of the mass flux pulsation in plane $\left(z^{\prime}, t\right)$ for the cross sections at $x=20,30$ and $40 \mathrm{~mm}$ are presented in Fig. 2. The measurements were provided in the area of the maximum level of the boundary layer pulsation. For each cross section the hot-wire probe was moved parallel to the leading edge and at a constant distance to the wall.
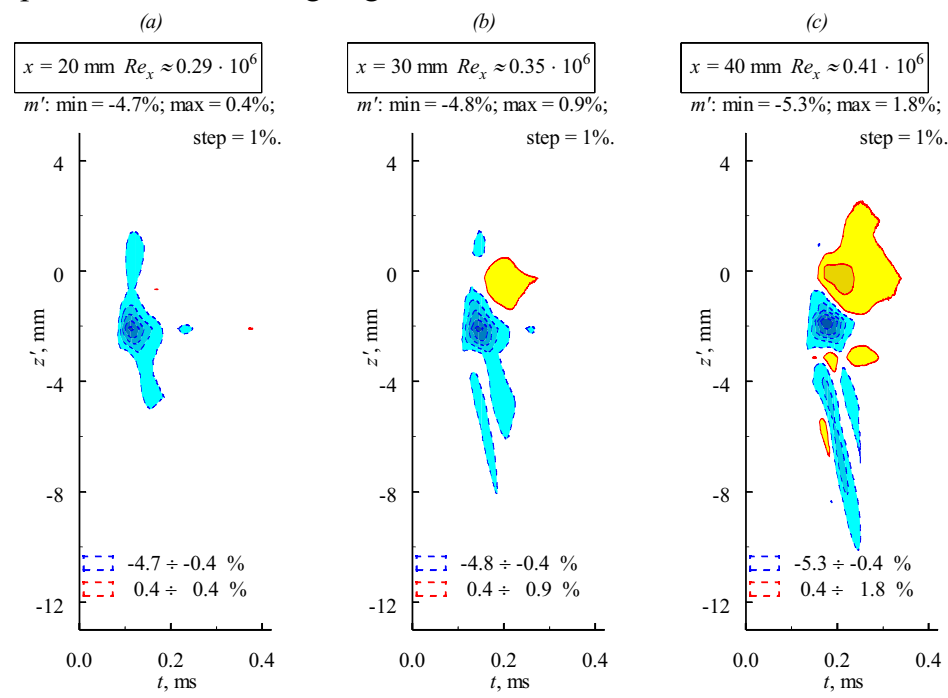

Fig. 2. Contour lines of the mass-flow pulsations of the wave packet in the plane $\left(z^{\prime}, t\right)$ at $x=20(a)$, $30(b)$ and $40(c) \mathrm{mm}$.

The disturbances exited by the discharge are asymmetric and shifted to negative values of the $z^{\prime}$-coordinate. This result differs from the case of a two-dimensional supersonic boundary layer. This is due to the crossflow in the boundary layer of the swept-wing. Similar results were obtained in the experiments on the evolution of localized disturbances in an incompressible boundary layer [20].

In the initial section, the perturbation from a pulsed discharge is a flow defect localized in space and time. The spatial scales of the wave packet increase downstream. The structures of excess and decrease of the mass flow form at the boundaries of the disturbance from the discharge. At $z^{\prime}=-3 \div 4 \mathrm{~mm}$ wave packet is represented as a vortex in the plane of the model surface. At $\mathrm{z}^{\prime}<-3 \mathrm{~mm}$ the short-term defects of mean flow are observed. 
Moments of time of the wave packet registration are varying downstream. Thus the velocity of propagation downstream of the wave packet can be calculated. It is estimated that the velocities of the wave packet propagation is about $0.5 \div 0.6 \cdot U_{\infty}$.

\section{Conclusion}

For the first time the evolution of a wave packet in a three-dimensional supersonic boundary layer has been experimentally investigated. The structure of the wave packet has been studied by the hot-wire measurements. It has been found that the structure of the wave packet in swept-wing boundary layer is not symmetric in contrast to the case of a twodimensional share layer. Also, the velocity of the propagation downstream of the wave packet has been estimated.

The work was financially supported by the Russian Science Foundation (Project No. 17-19-01289).

\section{References}

1. A.D. Kosinov, A.A. Maslov, S.G. Shevelkov, J. Fluid Mech. 219, 621 (1990)

2. Yu.G. Yermolaev, G.L. Kolosov, A.D. Kosinov, N.V. Semionov, Fluid Dynamics 49, $188(2014)$

3. A.D. Kosinov, G.L. Kolosov, N.V. Semionov, Yu.G. Yermolaev, Phys. Fluid, 28, 064101 (2016)

4. A.V. Panina, A.D. Kosinov, Y.G. Yermolaev, V.N. Gorev, N.V. Semionov, Thermophys. Aeromech. 21, 3 (2014)

5. A.N. Kudryavtsev, D.V. Khotyanovsky, Thermophys. Aeromech. 22, 559 (2015)

6. C.S.J. Mayer, S.Wernz, H.F. Fasel, J. Fluid Mech. 668, 113 (2011)

7. A.A. Maslov, A.N. Shiplyuk, D.A. Bountin, A.A. Sidorenko, J. Spacecraft Rockets 43, 71 (2006)

8. D.A. Bountin, A.N. Shiplyuk, A.A. Maslov, J. Fluid Mech. 611, 427 (2008)

9. A.A. Maslov, A.N. Shiplyuk, A.A. Sidorenko, D. Arnal, J. Fluid Mech. 426, 73 (2001)

10. M. Gaster, I. Grant, Proc. R. Soc. Lond., A 347, 253 (1975)

11. M. Gaster, Proc. R. Soc. Lond., A 347, 271 (1975)

12. M.M. Katasonov, S.H. Park, H.J. Sung, V.V. Kozlov, Exp. Fluids 38, 363 (2005)

13. K.M. Casper, S. J. Beresh, S. P. Schneider, J. Fluid Mech. 756, 1058 (2014)

14. C. S. J. Mayer, A.C. Laible, H.F. Fasel, AIAA J. 49, 67 (2011)

15. J. Sivasubramanian, H.F. Fasel, J. Fluid Mech. 756, 600-649 (2014)

16. A. Novikov, I. Egorov, A. Fedorov, AIAA J., 54, 2034-2050 (2016)

17. Y.G. Yermolaev, A.A. Yatskikh, A.D. Kosinov, N.V. Semionov, AIP Conference Proceedings, 1770, 030037 (2016)

18. A.A. Yatskikh, Y.G. Yermolaev, A.D. Kosinov, N.V. Semionov, Thermophys. Aeromech. 22, 17 (2015)

19. A.A. Yatskikh, Y.G. Yermolaev, A.D. Kosinov, N.V. Semionov, J. Visualization (2017) doi:10.1007/s12650-016-0414-2 (to be published)

20. G.R. Grek, M.M. Katasonov, V.V. Kozlov, Thermophys. Aeromech. 15, 549 (2008) 